\title{
Operaísmo y ecología-mundo.Por una teoría política de la crisis ecológica
}

\author{
EMANUELE LEONARDI*
}

\section{RESUMEN}

Este artículo pretende articular un encuentro entre el operaísmo y la ecología-mundo, es decir, un encuentro entre dos paradigmas teóricos cada vez más objeto de debate a nivel global, pero que, hasta el momento, no han sido analizados en estrecha relación. El operaísmo es una corriente del marxismo heterodoxo caracterizada por centrarse en la ambivalencia de la condición de la clase obrera (fuerza de trabajo/trabajo abstracto dentro del capital, clase obrera/trabajo vivo contra el capital) y la noción de la composición de clases sociales. La ecología-mundo puede definirse como un diálogo internacional que desarrolla el análisis del sistema-mundo desde una perspectiva ambiental: el capitalismo, por lo tanto, no tiene un régimen ecológico, sino que es un régimen ecológico, es decir, constituye un modo especifico de organizar la naturaleza. El objetivo de este artículo es demostrar que, a pesar de que las dos perspectivas se relacionan con la cuestión de la crisis (ecológica) de forma muy distinta, ellas pueden integrarse eficazmente si son yuxtapuestas a otro nivel: el del análisis histórico-político de la cuestión medioambiental. En su origen, ambos planteamientos revisan la teoría de la crisis de Marx, pero no eluden la polarización que caracteriza su evolución: mientras que el operaísmo tiende a reafirmar la tradición que considera la crisis como un momento de desarrollo, la ecología-mundo desarrolla la teoría de la brecha metabólica de un modo bastante inaudito. La convergencia entre estos dos paradigmas - lo que, en realidad, constituye un exigente intercambio teórico y que, por lo tanto, requiere una intensa reflexión por parte de ambas posiciones- puede producirse a través de una relectura del proceso histórico de la politización de la ecología. Aunque se suele situarlo entre mediados de los años setenta y la siguiente década - tras el gran ciclo de conflictos fordistas-, en los últimos años se está comprobando una hipótesis distinta: esta politización no sólo ocurrió una década antes, pero también, y, sobre todo, sucedió debido a, y no a pesar de, las luchas del movimiento obrero (en estrecha relación con el surgimiento del feminismo revolucionario). En ese contexto, la economía verde neoliberal es decir, el intento capitalista de internalizar el límite ecológico, transformándolo de un obstáculo a la valorización a una estrategia innovadora de acumulación - asume simultáneamente la forma de desarrollo (en línea con la hipótesis postulada por el operaísmo) y la de antidesarrollo (en línea con la hipótesis defendida por la ecología-mundo). De esta "convergencia" plausible podría emerger entonces una interpretación política de la crisis ecológica contemporánea capaz de cuestionar la relación entre el capitalismo y la naturaleza, al evitar tanto el catastrofismo como la afinidad electiva entre la lógica del beneficio y la lógica de la protección medioambiental..

\section{Palabras clave}

Operaísmo; ecología-mundo; teoría de la crisis; valor negativo; economía verde.

\section{TitLe}

Autonomist Marxism and World-Ecology. For a political theory of ecological crisis

\section{Extended Abstract}

The paper aims to articulate an "encounter" between Autonomist Marxism (AM) and World-Ecology (WE), that is, between two theoretical paradigms increasingly discussed at the global level, but so far never analyzed in close connection to one another.AM is a current of unorthodox Marxism that is characterized, methodologically, by the partiality of the point of view, the constitutive unity of thought and conflict, the ambivalence of the working-class condition (labor force / abstract labor within capital, working class / living labor against capital), and the centrality of class composition. Politically,AM proposes two

DOI:

https://doi.org//0.15366/relacionesinternacionales2021.47.004

Formato de citación recomendado:

LEONARDI, Emanuele (202I). “Operaísmo y ecología-mundo. Por una teoría política de la crisis ecológica”, Relaciones Internacionales, $\mathrm{n}^{\circ}$ 47, pp. 85-99.
* Emanuele LEONARDI, Profesor en Sociología en la Universidad de Parma (Italia) e investigador afiliado al Centro de Estudios Sociales de la Universidad de Coimbra (Portugal). Sus intereses de investigación incluyen: ecología política, movimientos de justicia climática y su crítica al comercio del carbono; ambientalismo de la clase trabajadora. Contacto: lele. leonardi@gmail.com

Recibido:

I 2.1 I.2020

Aceptado:

09.03.202।

Traducción: Fátima Patrícia OLIVEIRA 
main innovations: the practice of refusal of work, and the so-called Copernican revolution, according to which class struggle comes first and capitalist organization follows suit (instituting, therefore, a causal and incremental link between workers' unrest and capitalist development).

WE can be defined as a global conversation that develops the analysis of the world-system along distinctively environmental lines: capitalism, therefore, does not have an ecological regime, but rather is an ecological regime, i.e. a specific way of organizing nature. Beyond any residue of Cartesian dualism, the concept of world-ecology refers to an original mixture of social dynamics and natural elements that make up the capitalist mode of production in its historical development, and in its tendency to become a world-market. In this framework, the capitalist theory of value imposes space as flat and geometric, time as homogeneous and linear, and nature as external, infinite, and free.

The aim of this paper is to show that, although the two perspectives relate to the question of the (ecological) crisis in a very different way, they can be effectively integrated if juxtaposed on a different level - that of the historico-political analysis of the question concerning the environment. Both approaches originally rework Marx's crisis theory, but they do not completely avoid the polarization that marked its evolution: development vs. catastrophe.

AM tends to renew the tradition that sees the crisis as a moment of development and historicizes it through original interpretations of the cycle of struggles 1968-1973, claiming its defeat was "peculiar" as it imposed a change in the structure of capitalist valorization in the direction of an expansion of its accumulation base. The causes of this transition are to be found in the intersection between the financialization of the economy, the cognitization of labor and, above all, the becomingproductive of the sphere of social reproduction.

On the other hand, WE elaborates the so-called "breakdown" theory in unprecedented fashion. The starting point is a convincing reconstruction of the historical succession of long waves of economic cycles through an articulation of underproduction (of ecological surplus) and overproduction (of commodities). Thus,WE provides an instrumental ecological counterpoint to the socio-centric reading of AM through the fundamental notion of negative value - the most innovative analytical element with regard to the neoliberal form of crisis theory. However, the general discursive strategy follows that of every breakdown theory ever since the "classical" debate within the Second International.Therefore, it is aimed at showing that, although the crises of the twentieth century were developmental (that is, they fostered the capitalist restructuring at a higher level), the crisis we live through nowadays presents itself as epochal in that its result is deemed to be an inevitable collapse.

The convergence between the two paradigms - which is actually a rather demanding theoretical exchange, and as such require some deep rethinking for both positions - can take place through a re-reading of the historical process of politicization of ecology.Although it is customary to date it between the mid-seventies and the following decade -i.e. after the great cycle of Fordist conflicts - in recent years a different hypothesis is being tested: that such politicization occurred not only a decade earlier, but also, and above all, because of rather than despite the struggles of the workers' movement (in close connection with the rise cycle of revolutionary feminism). With particular regard to the Italian context, the struggles against noxiousness, which multiplied between the end of the 1960s and the beginning of the 1970s, and often in opposition to the confederal unions, were the first to fiercely criticize the so-called monetization of risk; that is,the idea that wage increases and/or organizational benefits could "compensate" for exposure to pollutants, even hazardous ones. Although this criticism would never become common sense of trade union action, such occurrence does not deny that it was first of all the strength of organized workers that blew up the compensatory mechanism and (im)posed the ecological question as politically unavoidable. Only at a later stage will the environmental movement emerge along with a new post-materialist sensitivity among the urbanized intermediate strata.

Against this background, the paper proposes an analysis of neoliberal green economy -i.e. the capitalist attempt to internalize the ecological limit, turning it from obstacle to valorization, through an innovative strategy of accumulationas simultaneously assuming the form of development (in accordance with AM hypothesis) and of anti-development (in accordance with WE hypothesis). From this plausible "convergence" could then emerge a political interpretation of the contemporary ecological crisis, capable of questioning the relationship between capitalism and nature by avoiding both catastrophism and the elective affinity between the logic of profit and the logic of environmental protection.

In this unprecedented context, WE can grasp the second aspect through the concept of negative value, which correctly conveys the message that climate change, health-related emergencies, and the narrowing of waste borders make the ecological crisis an unprecedented everyday reality in the history of capitalism. In fact, negative value implies an internal contradiction of the dynamics of capital and, above all, an ontological challenge to the valorization project, therefore to capitalist civilization tout court.

On the other hand, AM is in a privileged position to make sense of the shift from the rhetoric of limits to growth, which somehow alluded to environmental noxiousness as a crisis of capitalism, to a rhetoric of growth of limits, which identifies these latter as drivers of accumulation, as "filters" that turn the ecological constraint into a crisis for capitalism. Furthermore, AM can now show that commodities traded on environmental markets contain value as they are produced by hybrid units of labor (reproductive / informational) and nature (financialized). However, the developmental potential of such green economy must also be relativized. In fact, the process of enhancing the "free" activity of nature seems, at least until now, to be unable both to "repair" the environmental damage already done and to provide widespread social protections potentially able to compensate for the class polarization that invariably accompanies the multiplication of financial dividends. What neoliberal capitalism lacks is an inclusive mechanism capable of (partially) socializing financial profits either through a decarbonization of the economy, or through the formation of a new middle class (or both).

\section{KEYWORDS}

Autonomist marxism; world-ecology; crisis theory; negative value; green economy. 
Este artículo pretende articular un "encuentro" entre el operaísmo (Wright, 2017) y la ecología-mundo (Moore, 20I5a), es decir, entre dos paradigmas teóricos cada vez más objeto de debate a nivel global, pero que, hasta el momento, no han sido analizados en estrecha relación. El objetivo de este trabajo es demostrar que, a pesar de que las dos perspectivas se relacionan con la cuestión de la crisis (ecológica) de forma muy distinta, ellas pueden integrarse eficazmente si yuxtapuestas a otro nivel: el del análisis histórico-político de la cuestión medioambiental. De esta "convergencia" plausible - lo que, en realidad, constituye un exigente intercambio teórico y que, por lo tanto, implica una cierta renuncia de ambas partes- puede finalmente emerger una interpretación política de la crisis ecológica contemporánea. Esta interpretación es capaz de cuestionar la relación entre el capitalismo y la naturaleza, evitando los inconvenientes del catastrofismo y la afinidad electiva entre la lógica del beneficio y la lógica de la protección medioambiental.

Desde el punto de vista histórico, la formulación del operaísmo está estrechamente vinculada al ciclo de conflictos que sucedió en Italia en los años sesenta y setenta. En este contexto, cabrá que mencionar al menos algunas revistas y grupos políticos: Quaderni Rossi, Classe Operaia, y Rosso, por una parte; por otra, Potere Operaio y Autonomia Operaia. Desde una perspectiva metodológica, la propuesta operaísta se despliega en cuatro etapas: la parcialidad del punto de vista, la unión constitutiva del pensamiento y el conflicto, la ambivalencia de la condición de la clase obrera (fuerza de trabajo/trabajo abstracto dentro del capital, clase obrera/trabajo vivo contra el capital) y la centralidad de la noción de la composición de clases sociales (Filippini y Tomasello, 20 I0). Por último, desde el punto de vista político, las dos principales innovaciones del operaísmo consisten en el rechazo del trabajo, teorizado, entre otros, por Sergio Bologna y Antonio Negri sobre el cual se hablará más adelante con mayor detención-y la revolución copernicana, elaborada por Mario Tronti, según la cual primero ocurre la lucha de clases y la organización capitalista sigue su ejemplo (estableciendo, por consiguiente, un nexo causal y progresivo entre el malestar de los trabajadores y el desarrollo del capitalismo).

Finalmente, cabe destacar el modo específico como, a partir de los años noventa, un segundo momento del pensamiento operaísta "territorializa la filosofía contemporánea francesa en el campo del neomarxismo italiano [oltremarxismo]" (Chignola, 20I5, p. 32), en concreto la cuestión de la biopolítica planteada por Michel Foucault. Las herramientas analíticas foucaultianas, en particular la noción de gubernamentalidad neoliberal, son movilizadas para cuestionar la emergencia, partiendo de la crisis de los modelos de estado de bienestar socialdemócrata, de una nueva estrategia de acumulación capitalista basada en: la centralidad de la esfera de reproducción, la financiarización de la economía, y la cognitización del trabajo (Fumagalli et al., 2019). La encrucijada histórica donde el postoperaísmo entra en tensión con el concepto de crisis abre, por lo tanto, dos caminos: por una parte, el colapso de la dinámica social que ha sostenido los llamados "treinta gloriosos años" del capitalismo ( 1945-1975); por otra, los elementos de destrucción creativa de la sociedad que, a partir de los años ochenta, caracterizaron el proyecto hegemónico neoliberal. De paso, deberá mencionarse que estos caminos, y la relevancia de criticarlos desde una perspectiva ecológica, han sido analizados detenidamente por las más atentas corrientes de la ecología política (Pellizzoni, 2019). 
Con relación a la ecología-mundo, la referencia fundamental de este diálogo global (Leonardi y Pellizzoni, 2019) es Jason Moore, un sociólogo marxista conocido, sobre todo, por haber desarrollado un planteamiento del sistema-mundo propuesto por Giovanni Arrighi y Immanuel Wallerstein orientado hacia un enfoque riguroso en las cuestiones ambientales. Esto condujo a la formulación de la ecología-mundo, una propuesta según la cual el capitalismo no tiene un régimen ecológico, sino que es en sí mismo un régimen ecológico, es decir, una forma especifica de organizar la naturaleza. Más allá de algún remanente de dualismo cartesiano, el concepto de ecología-mundo se refiere a la amalgama original de las dinámicas sociales y los elementos naturales que constituyen el modelo de producción capitalista en su evolución histórica, en su tendencia para convertirse en un mercado mundial. En este marco, la teoría capitalista del valor considera el espacio plano y geométrico, el tiempo homogéneo y lineal, y la naturaleza externa, infinita y gratuita.

En concreto, la noción de naturaleza social abstracta nos permite comprender mejor los términos específicos mediante los cuales la naturaleza es internalizada durante el proceso de valoración como un límite habilitante, aunque invisible -o sea, constituye una condición indispensable para que el capital y el trabajo asalariado se acerquen, pero no es un factor directamente involucrado en el acto de creación de valor. Moore identifica la transición histórica de la propiedad de la tierra al trabajo asalariado como la fuente principal de productividad, que ocurrió a lo largo del siglo XVI, como la condición sine qua non para la internacionalización de la naturaleza en el valor. ¿Qué significa esto? Esto significa que, para que el proceso de valorización se produzca, las actividades vitales de las que la naturaleza es manifestación deberán ser transformadas de modo a que se ajusten a la lógica del valor. De modo resumido, podemos sintetizar el marco que de aquí emerge de la siguiente forma: el trabajo social abstracto —es decir, el trabajo asalariado organizado por el capital y medido en unidades de tiempo de trabajo- constituye la única fuente de valor en la esfera de producción. Sin embargo, para que los mecanismos de creación de valor (definidos por Moore como la zona de mercantilización o acumulación por capitalización) se pongan en marcha, es necesario que un gran volumen de trabajo no remunerado esté a la disposición del capital. Moore denomina este movimiento acumulación por apropiación, concepto que define el ámbito de la naturaleza social abstracta en la que los elementos tradicionalmente relegados a la esfera de reproducción (las tareas domésticas, el trabajo esclavo, los regalos gratuitos de la naturaleza) convergen. Estos elementos de reproducción - merece la pena reiterar- pueden funcionar como condición para el valor desde que se reconozcan como infinitos y gratuitos (nuevamente, habilitantes, aunque invisibles). En suma:

"Las técnicas capitalistas buscan movilizar y apropiarse de 'las fuerzas de la naturaleza' —impagadas - tanto como hacer productivas las 'fuerzas de trabajo' — pagadas - en su versión moderna —la producción de excedentes-. Este es el significado de la producción de naturaleza; la naturaleza no es un objeto preformado para el capital, sino una red de relaciones que el capital reformula para avanzar en las contribuciones de 'trabajo' biosférico no remunerado para la acumulación de capital. El capital, al hacer esto, es reformulado por la naturaleza como conjunto" (Moore, 20 I4, p. 295).

Un buen ejemplo de la estrategia argumentativa de Moore es representado por el carbón: a 
través de la ecología-mundo es posible reconstruir con exactitud cómo las relaciones sociales que emergieron a partir del siglo XVI transformaron el carbón de un simple mineral a un combustible fósil, así como definieron el conjunto de conocimientos biológicos, físicos y geológicos necesarios para hacer concebible y utilizable el concepto de "combustible". De ello se desprende que el desarrollo de la producción sería inconcebible al margen de las relaciones de valor establecidas en la modernidad temprana:"el prejuicio del materialismo verde nos dice que 'el carbón ha cambiado el mundo’. ¿Pero no será la formulación contraria más plausible? Las nuevas relaciones mercantiles han transformado el carbón (al mismo tiempo que impulsaron su poder histórico)" (Moore, 2017, Pp. 53-54).

\section{I. ¿Colapso o desarrollo? Lecturas contrapuestas de la teoría de la crisis (partiendo de Marx)}

A pesar de que tanto el operaísmo como la ecología-mundo establecen una relación constitutiva con el pensamiento de Marx, las conclusiones a que llegan, particularmente con respecto al concepto de crisis, son divergentes. Al fin y al cabo, el propio pensamiento de Marx en este sentido es sencillo y problemático a la vez. Sencillo porque no cabe duda de que la crisis pone de manifiesto las contradicciones capitalistas y los límites intrínsecos del desarrollo del capital. Problemático porque coexiste una pluralidad de perspectivas distintas, de las que cabe destacar por lo menos tres: la crisis del agotamiento del ejército industrial de reserva, la crisis de sobreproducción y la crisis de realización (Bellofiore, 2012). Además, estas crisis asientan sobre una tensión irresoluble y fundacional entre el elemento revolucionario de análisis - que busca derrocar a la sociedad burguesa - y el elemento científico — que, en cambio, tiene como objetivo describir con exactitud el funcionamiento del sistema (Colletti, 1970). Resumiendo en pocas líneas este largo debate, es posible afirmar que un punto en común de todos los marxismos reside en las características necesarias de la crisis para las dinámicas expansivas del capitalismo —que surgen precisamente como un modo de producción inestable, "forzado" a partir de una lógica de hierro interna para revolucionar y ampliar contantemente la basis de su propia acumulación-, mientras que las discrepancias se centran tanto en las causas económicas (ioferta excesiva o demanda insuficiente?) como en los resultados políticos (icolapso o desarrollo?).

En la comparación entre el operaísmo y la ecología-mundo que propongo en este artículo, la atención se centra en el segundo tema de la disputa. El primer operaísmo y los análisis subsecuentes sobre la producción biopolítica, en la cual la completa subsunción de la sociedad (no del trabajo) sería alcanzada, consideran la crisis como un factor de desarrollo (inicialmente) y de gobernanza del desarrollo (posteriormente). Al fin y al cabo, esta es una conclusión coherente a la que llegan tanto la revolución copernicana de Mario Tronti — primero, la lucha de clases y luego la reestructuración capitalista - como la inversión foucaultiana de la relación entre el poder y la resistencia, donde esta última actúa como una fuerza afirmativa y el anterior como una fuerza reactiva.Ya en una carta de Tronti para Raniero Panzieri de fecha 30 de junio de I96I, poder leerse:

"El problema del desarrollo capitalista no puede desvincularse del problema de las crisis. Por este hecho - el hecho de que la crisis es un momento del desarrollo capitalista- - [...], el análisis de la crisis sólo puede ser un análisis dinámico del sistema, es 
decir, un análisis de las dinámicas del sistema. Cuanto más se reflexiona sobre ello, y cuanto más uno se convence de que el problema a ser resulto reside aquí —en esta compleja unión entre el desarrollo capitalista y la revolución obrera- [...], es justamente de esta unión material que debe nacer el nuevo experimento socialista" (Tronti, 196I, como se citó en Trotta y Milana, 2008, p. I 18)'.

El núcleo central del análisis de Tronti es planteado y ampliado por Negri en un ensayo escrito en 1968, titulado Marx on Cycle and Crisis. La excepcionalidad de la reinterpretación de la teoría de la crisis propuesta por Negri puede inferirse a través de los comentarios admirativos de un crítico implacable como Riccardo Bellofiore, quien, a principio, la definió un análisis "con una profundidad e influencia notables" (1982, p. 102) y, después, "genial a su manera" (2008, p. 299). Fundamentalmente, Negri cree que la reflexión de Marx es adecuada al capitalismo del siglo XIX. Simultáneamente, el horizonte de los años sesenta está caracterizado por la "completa reestructuración de las relaciones de poder" impuesta por la Revolución de Octubre, a la cual el capital colectivo había respondido anteriormente con el New Deal, es decir, con la integración de la clase obrera en el mecanismo de desarrollo capitalista, $y$, después, con el pacto fordista consagrado en el estado de bienestar (1972, p. 199). En este contexto, la crisis deja de ser un elemento antagónico del desarrollo; más bien, se presenta como su elemento esencial. De hecho, es solo a través de la crisis "que el beneficio surge como un elemento del capitalismo, la verdadera cara del funcionamiento de la ley de valor", y es solo el discurso sobre la crisis y la necesidad que "proporciona al sistema un elemento de comprensión profunda de la realidad” (1972, p. 202). Sobre esta premisa, y más allá del New Deal (esto es, más allá de la perspectiva de Tronti), Negri se centra en la configuración completamente fordista del nexo crisis/desarrollo, recurriendo a John Maynard Keynes y, sobre todo, Joseph Alois Schumpeter:

"En Keynes el momento central de análisis es aquel que concibe el desarrollo como ritmo agregativo de las fuerzas de la producción social; en Schumpeter el momento central es el del descubrimiento de que un equilibrio agregativo de esta índole es un límite abstracto y que, por esta razón, la agregación puede darse sólo a condición de que sea una relación continua con una desagregación y una reforma continua del proceso. En Keynes, el desarrollo pretende ser alternativo a la crisis, en Schumpeter el desarrollo es una nueva figura completa del ciclo, y de este modo incluye la crisis y la utiliza en función del movimiento cíclico. De cualquier manera, los dos momentos son complementarios: se integran en el conocimiento de la necesidad del uso de la presión masificada de la clase obrera, de su control y rígida contención en las murallas del proceso dinámico del desarrollo.Ambos suministran singular claridad a la práctica capitalista más reciente" (1972, p. 203).

\footnotetext{
Agradezco a Michele Filippini por haberme informado de la existencia de esta carta.
} 
El rechazo categórico de cualquier teoría del colapso y el intento de comprender el concepto de crisis partiendo de la coyuntura histórica puede encontrarse en el análisis del colapso financiero de 2007-2008 propuesto por el operaísmo. En particular, cabe señalar tres aspectos significativos. En primer lugar, constituye un nuevo tipo de crisis: a pesar de que la financiarización no es en ningún caso un fenómeno nuevo (por ejemplo, los artículos de Marx para el Daily Tribune de Nueva York, en los últimos años cincuenta del siglo XIX, ofrecen un excelente análisis de la especulación financiera), su generalización y centralidad contemporáneas hacen obsoleta la oposición entre la economía real y la economía financiera. Esto no significa que la última absorbe la primera. Sino más bien indica que estos dos elementos deben ser concebidos como distintos pero inseparables. No son iguales, pero al margen de su vinculación pierden su significado como categorías interpretativas (Mezzadra y Fumagalli, 2009). En segundo lugar, las finanzas desempeñan un papel productivo y no un rol parasitario: "Esta crisis no se debe tan solo a la locura financiera. En cambio, debe entenderse partiendo de las especificidades del régimen de acumulación existente" (Lucarelli, 2009, p. I0I). Las finanzas participan directa y activamente en la producción de la plusvalía y, en consecuencia, la crisis es, en esencia, real y financiera. En tercer lugar, las finanzas son la piedra angular de la gubernamentalidad neoliberal: para que los mercados financieros gestionen la producción de plusvalía a nivel global, cada vez más sectores de la población deben depender directamente de ellos. Esta dependencia se manifiesta y actúa mediante varias formas de crédito, seguridad social y efectos sobre la riqueza. En consecuencia, "la financiarización [es] la forma actual de dominio capitalista" (Negri, 2009, p. 23I).

Más de diez años tras la explosión de la burbuja inmobiliaria, y en la ausencia de señales de recuperación económica, el postoperaísmo mantiene la preeminencia del vínculo entre la crisis y el desarrollo, pero lo articula alrededor de dos polos argumentativos distintos. El primero, propuesto por Dario Gentili (20I8), recupera el concepto gramsciano de interregnum para demostrar que los escollos de la dinámica del capitalismo biopolítico no están destinados a solventarse, sino que deben seguir indefinidamente en una especie de limbo entre la vida y la muerte. El segundo, formulado por Negri y Michael Hardt, propone de nuevo la actualidad del elemento de ruptura política (protagonizada ya no por la clase obrera, sino por la multitud), arguyendo que "los antagonismos constitutivos de la producción social, cooperativa y cognitiva se producen en el marco del capital financiero y atacan el núcleo de los mecanismos extractivos" (2018, p. 269). Además, no debe subestimarse el enorme impacto ambiental generado por el régimen de acumulación impulsado por el sector financiero: un ejemplo paradigmático, pero ciertamente no el único, de ello es la relación constitutiva que vincula la especulación financiera y el acaparamiento de tierras (Fairbarn, 20I4; Benegiamo, 202I).

En cuanto a la ecología-mundo, su contribución fundamental es poner de relieve la dimensión ecológica de la teoría de la crisis. En primer lugar, Moore rastrea en el legado de Marx el análisis de un bloqueo ambiental en la dinámica capitalista: la crisis de subproducción (de "naturaleza generosa y gratuita", recordando las palabras de Ricardo). Teniendo en cuenta la necesidad de la acumulación de incorporar una creciente cantidad de actividad natural barata y trabajo humano no remunerado para mantener el "supuesto estructural del incremento en la composición del valor del capital" (Avallone, 2015, p. 19), se desprende que cada ciclo de desarrollo se enfrenta a una "tendencia a caer en el excedente ecológico" (Moore, 2015a, p. 108), es decir, la ratio entre la masa total del capital y la parte de la naturaleza y el trabajo gratuito. Esto depende del 
hecho de que "el porcentaje de beneficio aumenta o disminuye en proporción inversa al precio de las materias primas" (Marx, 1973, p. 146), que tiende a aumentar como la disponibilidad de la naturaleza gratuita y el trabajo disminuye debido a la aceleración de las tasas de acumulación. Por tanto, la crisis de subproducción (de una naturaleza "gratuita") pone de manifiesto la dimensión ecológica de la crisis de sobreproducción (de productos).

En segundo lugar, la ecología-mundo proporciona una reconstrucción convincente de la sucesión histórica de los largos ciclos económicos precisamente a través de la articulación que se establece entre la subproducción y la sobreproducción. No disponemos de espacio en este artículo para analizar la transición del siglo XVI holandés al largo siglo XVIII británico y cómo triunfó la hegemonía estadounidense a lo largo del siglo XX. Sin embargo, es posible centrarnos en el último ciclo de producción de naturaleza barata y el agotamiento de la naturaleza. Es nos permite, por una parte, reconocer la identidad sustancial de la periodización a través del enfoque del operaísmo, y, por otra, destacar la persistencia de una actitud fatalista en la ecología-mundo, si bien muy distinta de la llamada "clásica" (a saber, situada en el debate de la Segunda Internacional, entre los siglos XIX y XX). Con relación al primer aspecto, Moore sitúa en la primera mitad de los años treinta, en los Estados Unidos —es decir, en el marco del New Deal- las causas desencadenantes de la Revolución Verde como proceso de innovación de las prácticas agrícolas (basadas principalmente en el uso generalizado de pesticidas y fertilizantes industriales), que condujo a un incremento significativo de la producción agrícola entre los años cuarenta y los años setenta ${ }^{2}$. A partir de la declaración de inconvertibilidad de dólar por Nixon en 1971, y la primera crisis del petróleo en 1973 —dos acontecimientos clave en la interpretación operaísta del declive del fordismo-, se estancó la productividad agrícola y con ella la producción de naturaleza barata. Según el análisis de Moore, el discurso sobre las biotecnologías verdes, que habría respaldado la incorporación financiera de los productos alimenticios, adolece de incoherencia con la crisis de 2003 (un pariente muy cercano del colapso del sector puntocom en 200I; ambos entrarían en la gran recesión que empezó en 2007-2008). También en este caso coincide las periodizaciones del operaísmo y la ecología-mundo.Además, la ecología-mundo faculta un contrapunto ecológico instrumental para la lectura sociocéntrica del operaísmo a través del concepto fundamental de valor negativo —el elemento más innovador del análisis propuesto por Moore relativamente a la forma neoliberal de la teoría de la crisis.Abordando "la transición de la plusvalía al valor negativo", Moore observa que:

"En esta transición, las 'viejas' contradicciones del agotamiento de la naturaleza se están encontrando con las 'nuevas' contradicciones de los desechos y la toxificación ambiental. El viejo modelo productivista, la ley de la naturaleza barata, ha sabido encontrar soluciones para el agotamiento de recursos. Pero no está adaptado para lidiar con el valor negativo, es decir, aquellas formas de la naturaleza que eluden y socavan los parches de la naturaleza barata. Las supermalezas son un ejemplo expresivo de esta tendencia. Ahora solo pueden

\footnotetext{
Harry Cleaver argumenta de forma convincente que la Revolución Verde debe interpretarse como "un intento en el periodo de la posguerra por contener las revoluciones sociales y proteger la rentabilidad mundial” (1972, p. 177). Por otras palabras, Cleaver plantea la hipótesis de la existencia de un estrecho vínculo entre el incremento global de la productividad agrícola debido a los avances tecnológicos y la política exterior anticomunista de Estados Unidos. Por consiguiente, semillas más productivas constituyen aparentemente un arma estratégica en la Guerra Fría, es decir, un contrapunto al espectro de las revueltas en Asia y África.
} 
controlarse con una mayor toxificación del medioambiente y mayores gastos. Por otra parte, la toxificación directa e indirecta del medioambiente contribuyen con mayor fuerza para nuevas formas de valor negativo: el cambio climático, epidemias de cáncer y otros" (Moore, 20I5, pp. 274-275).

Tras establecer un punto de contacto entre estos dos enfoques con respecto a la periodización que proponen, ha llegado el momento de evidenciar una importante divergencia que se refiere directamente a la cuestión del desenlace de la crisis, es decir, la alternativa colapsodesarrollo. A pesar de sui generis, me parece que el análisis de Moore replantea los elementos esenciales del debate sobre el colapso; dos autores que resuenan en las obras de la ecologíamundo son Rosa Luxemburg (20I2) y Heryk Grossman (20I0). Es cierto que Moore no recurre al estilo clásico de la "versión roja-verde de la teoría del colapso", cuya componente fundamental es la sustitución de los límites físicos de la anarquía del mercado como una contradicción incorrigible del capitalismo (Bellofiore, 1988, p. 21). En este análisis, la cuestión de la finitud ambiental no se presenta como una "segunda" contradicción, es decir, paralelamente a la "primera", entre el capital y el trabajo asalariado (O’Connor, 1988), ni como una acumulación de catástrofe (Bellamy Foster, $20 \mathrm{II}$ ). Por el contrario, surge como una variable dependente de la producción de la naturaleza externa, infinita y gratuita. Sin embargo, la estrategia discursiva sigue la de todas las teorías del colapso desde el debate clásico. Por consiguiente, tiene como objetivo demostrar que, a pesar de que las crisis a lo largo del siglo $X X$ fueron desarrollistas (o sea, fomentaron la reestructuración capitalista a un nivel superior), la crisis que estamos atravesando actualmente su presenta como epocal porque su efecto se considera ser un colapso inevitable. Esta postura es evidente tanto en la identificación de una forma de crisis de subproducción sin precedentes, que en el adviento del neoliberalismo se convierte en la "ley general de sobrepolución" (Moore, 20 I 5b, p. 27I) —es decir, la tendencia para agotar más rápidamente las fronteras de desechos ${ }^{3}$ - como en la frecuencia de declaraciones algo apocalípticas como:

"El cambio hacia la financiarización y una intensificación de las relaciones mercantiles en la esfera de reproducción ha sido una poderosa forma de posponer el inevitable retroceso de la estrategia de la naturaleza barata de la modernidad. Ha permitido al capitalismo sobrevivir. Pero ipor cuánto tiempo más?" (20I5, p. 305).

\section{La genealogía obrera de la cuestión medioambiental y la centralidad política de la crisis ecológica}

Hasta el momento, he intentado describir el solapamiento entre los enfoques del operaísmo y la ecología-mundo, en el que el primero proporcionar una configuración precisa del cuerno social de la teoría de la crisis mientras que el segundo pone de manifiesto su dimensión ambiental subyacente. Este constituye, de hecho, un primer paso analítico fundamental, ya que encarna el elemento antagónico fruto de las luchas, crucial para el operaísmo, y la perspectiva capitalocéntrica de la ecología-mundo. Se propone conciliar los aspectos subjetivos y objetivos de modo a hacerse

\footnotetext{
Sobre este tema, véase el brillante trabajo de Armiero (2021).
} 
una valoración del capitalismo contemporáneo, asumiendo la posibilidad de un colapso y la de una reorganización imprevisible, sin dar por sentada ninguna de las opciones. No obstante, mi argumentación exige un paso más, una vez que la mezcla de enfoques alternativos al mismo nivel analítico representaría una contradicción. Por tanto, se enmarca la articulación entre el operaísmo y la ecología-mundo siguiendo líneas discursivas distintas, pero que se intersecan -líneas que, para rastrearse, requieren un reconocimiento previo de la no autosuficiencia de ambos enfoques y una renunciación mutua de algunas asunciones fundacionales-.

En primer lugar, es necesario cuestionar cómo la crisis ecológica se convierte, entre los años sesenta y setenta, en un tema político en sentido estricto, es decir, ineludible para todos los actores implicados y a todas las escalas. Es importante aquí hacer una distinción entre la degradación medioambiental, cuyos ejemplos pueden encontrarse en todas las épocas y sociedades, y la crisis ecológica, cuya causa directa radica en el modo capitalista de organizar el trabajo, dependiendo de la necesidad de acumulación y crecimiento que caracteriza el interés económico. En este contexto, es necesario concretar más: aunque es habitual fechar la politización generalizada de las cuestiones ambientales a mediados de los años setenta y en la década siguiente (Della Valentina, 201 I) —es decir, tras el gran ciclo de conflictos fordistas - , en los últimos años, se está intentando demostrar una hipótesis distinta y más radical. Desde esta perspectiva, esta politización tuvo lugar no sólo una década antes, sino que también, y sobre todo, ocurrió debido a, y no a pesar de, las luchas del movimiento obrero (Leonardi, 2017 $7^{\text {a }}$, tanto el "oficial” como el "revolucionario" (Rector, 20I4; Citoni y Papa, 2017; Davigo, 2017). Stefania Barca sugiere el término "ambientalismo obrero" para describir la formulación de un conocimiento sectario sobre el ambiente laboral que se niega a ser ignorado o a dejarse intimidar: "El lugar de trabajo era considerado un tipo particular de ecosistema, y el trabajador era quien lo conocía mejor" (Barca, 201 I, p. 103; Barca, 2020). De hecho, las luchas contra la nocividad ${ }^{4}$, que se multiplicaron a finales de los años sesenta y comienzos de los años setenta, a menudo en oposición a los sindicatos confederales, son las primeras a criticar la llamada monetización del riesgo, es decir, la idea de que los aumentos salariales y/o los beneficios institucionales podrían compensar la exposición a sustancias contaminantes, incluso a sustancias peligrosas (Milanaccio y Ricolfi, I 976; Sacchetto y Sbrogiò, 2009).

Por supuesto, esta crítica nunca formará parte del sentido común de la acción sindical, cuyo legado a finales de los años setenta se considera "bastante negativo", puesto que "la aplicación de medidas de seguridad ligeramente mejores" estaba "subordinada a las políticas corporativas implementadas periódicamente" (Davigo, 20 I7, p. 176). Pero esto no niega el hecho de que fue, ante todo, la fuerza de los trabajadores organizados que desintegró estos mecanismos compensatorios y planteó la inevitable cuestión ecológica. Sólo en una fase posterior emergerá el movimiento ambientalista junto con una nueva sensibilidad postmaterialista entre los estratos urbanos intermedios (Inglehart, 1997).

Cabe añadir a este panorama dos elementos importantes. En primer lugar, las luchas contra la nocividad que tuvieron lugar entre 1968 y 1973 (ubicadas en el marco del trabajo social abstracto) no habrían tenido el impacto disruptivo que, de hecho, tuvieron si no estuvieran

4 El autor emplea el término inglés noxiousness [N. de la T.]. Lorenzo Feltrin y Devi Sacchetto (202I) usan el término noxiousness para traducir la palabra italiana nocività, que "se refiere a la propiedad de causar daño. Su empleo por el movimiento obrero llevó a que su significado se ampliara de modo a abarcar el daño infringido sobre la vida humana y la vida no humana, por lo que no puede traducirse como 'daño a la salud (humana)' ni como 'degradación medioambiental (no humana)'”. 
vinculadas a conflictos más amplios que, en ese periodo, legitimaron la centralidad inaudita de los sujetos en las esferas de reproducción social (que se sitúan en el marco de la naturaleza social abstracta). El feminismo, los movimientos de descolonización y el ambientalismo comunitario están excluidos del estado de bienestar socialdemócrata que transpuso a nivel político -el de las luchas cualitativas - el potencial político de la crisis ecológica revelado por el movimiento obrero. No obstante, este movimiento no logró elaborar una estrategia unificada al respecto: más bien, surge una tensión entre las perspectivas de la liberación en el trabajo -apoyada por Bruno Trentin, el secretario de la Federación Italiana de Trabajadores Metalúrgicos (FIOM, según el acrónimo italiano), y por sectores del Partido Comunista Italiano- y las perspectivas de la liberación del trabajo, concretamente la ambición de uno liberarse del trabajo, —respaldadas por grupos obreros organizados (Potere Operaio, en un primer momento, y, posteriormente, Autonomia Operaia) - Ambas formulaciones expanden su análisis más allá del ámbito salarial, por lo que se configuran como críticas a la lógica de valor (de cambio) —en las palabras de Karl Heinz Roth, operan "traspasando las luchas por un aumento salarial” (2009, p. I52). No obstante, estas perspectivas poseen horizontes distintos y que no son fácilmente conciliables. La primera considera que es factible, por así decirlo, recuperar el trabajo asalariado en nombre del trabajo en un sentido genérico, como una actividad humana en la cual el individuo expresa su auténtica personalidad. Se trataría de proceder a una desalienación del trabajo asalariado mediante el control del proceso de producción por los trabajadores para que cada uno pueda desarrollar eventualmente su singularidad (Barca, 2017). Por otra parte, la segunda perspectiva preconiza el rechazo del trabajo (asalariado) como una actividad impuesta por el capital. Este rechazo no pretende tan sólo presentar una crítica radical del trabajo asalariado (la fatiga laboral, el trabajo/empleo, el trabajo expropiado), sino no que se propone a abolir el valor y reafirmar la lógica de la riqueza (basada en el valor de uso) a través del empleo de consignas como "reducción de la jornada laboral", "reducción de los ritmos", "rechazo de la nocividad en favor del derecho a la salud" y "sueldos iguales para todos" (y desvinculados de la productividad).

Según Nanni Balestrini y Primo Moroni (2005), la incapacidad de reconciliar estas dos opciones $-\mathrm{y}$, añado, de vincularlas más estrechamente con las luchas de los sujetos de reproducción - condujo al fracaso del ciclo de luchas sucedido entre 1968 y 1973. En lugar del poder obrero sobre la composición cualitativa de la producción, se manifestó la violenta reacción del capital: el desmantelamiento del trabajo (y sus organizaciones), el desmantelamiento del estado de bienestar y la financiarización acelerada. Sin embargo, cabe señalar que el fracaso de la "época de movimientos" fue peculiar. En efecto, estas luchas han sido impulsadas por el cambio en la estructura de valorización capitalista hacia la ampliación de su basis de acumulación. Las causas de esta transición residen en la intersección entre la financiarización de la economía, la cognitización del trabajo y, sobre todo, la orientación hacia la productividad en la esfera de reproducción social. En otras palabras, la transición debe considerarse un esfuerzo del capital por convertir su propio obstáculo en una fuerza motriz. De hecho, ¿qué es la economía verde sino un intento de internalizar la limitación medioambiental y transformar a ese obstáculo en una oportunidad de negocio a través de la creación de mercados ad hoc? Reiterando, la economía verde postula una afinidad electiva entre la lógica del beneficio y la lógica de la protección ambiental, y este es el núcleo de la "revolución desde arriba" puesta en marcha ${ }^{5}$. El historiador del movimiento obrero 5 Otra forma de decirlo sería: el ciclo de luchas sucedido entre 1968 y 1973, liderado por la reproducción social, impuso una bifurcación del
nexo valor/naturaleza: mientras que, antes, “la naturaleza" se consideraba infinita y gratuita (el nexo valor/naturaleza "clásico"), después del 
Sergio Bologna comprendió este elemento fundamental en 1988; no fue más que una intuición, seguramente, pero una muy relevante, principalmente si vista en retrospectiva. En un artículo para Primo Maggio, escribe:"El capital necesita del ambientalismo para alcanzar la frontera de una nueva revolución industrial” (I988, p.8).

Sin embargo, esto no significa que la crisis ha sido resuelta sin conflictos por una etapa superior del desarrollo capitalista. Si fuera el caso, la economía verde funcionaría perfectamente, mientras que todos los datos disponibles, incluso los de sus defensores más entusiastas, demuestran lo contrario (Ronchi, 20 I8): una clara contradicción entre la (supuesta) meta ecológica y los (reales) medios económicos de los mercados ambientales. En efecto, aunque la situación ecológica no ha mejorado, se ha creado una gran cantidad de valor, posteriormente transferido para empresas que hacen un uso intensivo de los combustibles fósiles mediante la producción de lo que podemos denominar "renta climática". Seguramente, para resolver este impase, podríamos argumentar que sería suficiente reverter los términos de esta contradicción y, por tanto, privilegiar la meta ecológica en detrimento de los medios económicos. Pero es justamente en este punto que podemos comprender la tensión irremediable en la que se encuentra la economía verde. Para que tenga éxito, debe renunciar al crecimiento/acumulación (la reproducción/la orientación hacia la productividad no ser pueden concebidos como subordinados, infinitos y gratuitos). No obstante, una vez que asume la acumulación/crecimiento como su propia razón de ser, la economía verde no puede permitirse hacerlo. Por tanto, parece razonable afirmar que, en el ámbito del régimen de acumulación dirigido por las finanzas, dependiente de la infraestructura digital y caracterizado por una orientación hacia la productividad, la importancia política de la crisis ecológica es un hecho indiscutible (Torre, 2020).

\section{Consideraciones finales: una nueva perspectiva basada en el operaísmo y la ecología- mundo}

En este contexto, en este artículo se sugiere un encuentro más profundo entre el operaísmo y la ecología-mundo. La crisis actual, la primera en la que la cuestión ecológica asume plenamente un significado político, se presenta simultáneamente como desarrollo y antidesarrollo. Moore capta claramente el segundo aspecto a través del concepto de valor negativo, expresando que el cambio

ciclo de luchas, ha empezado a verse también como un elemento directo de valorización (el "nuevo" nexo valor/naturaleza). Cabe destacar que este "nuevo" nexo no sustituye el "clásico", sino que lo complementa: es por ello que el conflicto interno entre el capital "marrón" y el capital "verde" no constituye una cortina de humo ideológica, sino que es una batalla urgente y actual.

6 Mi perspectiva sobre la "renta climática" difiere de otros enfoques detallados, tales como el de Felli (20I4) y el de Andreucci et al. (20I7). Felli arguye que los bonos y permisos de carbono no deben ser considerados mercancías, dado que no cristalizan tiempo de trabajo socialmente necesario. Por consiguiente, el comercio de carbono no podría constituir una nueva estrategia de acumulación, ya que las pseudomercacías intercambiadas en el ámbito de esa estrategia serían meramente permisos para emitir gases de efecto invernadero. Como tal, constituirían componentes esenciales de la "renta climática", siendo que la renta se definiría como "una relación distributiva —y no productiva - que juega un papel contradictorio en la dinámica del capital Andreucci et al., 20I7, p. 8).Además de considerar el medioambiente "gratuito" e "infinito", el capitalismo contemporáneo postula "la naturaleza" como un elemento interno de los circuitos de valoración.

En mi opinión, los tres procesos descritos anteriormente - la explotación de la reproducción social, la financiarización y la cognitización/ digitalización - han producido una bifurcación (no un agotamiento) de la teoría del valor. En consecuencia, las mercancías de carbono encierran trabajo social abstracto; de forma más sencilla, este trabajo es irreductible al tiempo cronológico, entendido como unidad de medida. Las mercancías de carbono deberían considerarse conjuntos de trabajo-naturaleza vinculados por información y explotados por la lógica del mercado. El valor generado por los bonos de carbono no procede de los árboles o los océanos, sino más bien de su potencial de hundimiento calculado políticamente para ajustarse a las estrategias de los mercados financieros; no de una semilla, sino de una secuencia genética que los hace resistentes a los pesticidas biotecnológicos. 
climático, las emergencias sanitarias y el estrechamiento de las fronteras de desechos hacen de la crisis ecológica una realidad cuotidiana sin precedentes en la historia del capitalismo. En efecto, el valor negativo supone una contradicción en la dinámica del capital $y$, sobre todo, un reto ontológico al proyecto de valoración, y, por consiguiente, a la "civilización capitalista" (20I5b, p. 278). Sin embargo, Moore se equivoca al considerar este impase necesario: "el trabajo no remunerado puede ser —y frecuentemente es— medido (en los 'servicios ecosistémicos'); pero no puede ser valorado" (p. 300).

La cuestión a que el operaísmo no contesta, y que en mi opinión puede ser comprendida a través de la ecología-mundo, es que la lógica del valor no está en absoluto "dependiente del dualismo" (2015b, p. 292). El hecho de que, hasta los años setenta, la acumulación de capital asentó sobre la separación "ficticia" entre la sociedad interna y la naturaleza externa (infinita y gratuita) no implica que dependa de ese carácter dualista de la realidad para operar. Centrándose en las investigaciones más novedosas sobre la economía verde, Luigi Pellizzoni ha demostrado adecuadamente cómo esta indeterminación postdualista que caracteriza la geoingeniería y las biotecnologías no representa un obstáculo a la utilización del capital; más bien, actúa como premisa de un "nuevo dominio sobre la naturaleza" (2019, p. II) ${ }^{7}$. Por otras palabras, existe un desplazamiento de la retórica de los límites al crecimiento, que, de algún modo, hace referencia a la nocividad ambiental como una crisis del capitalismo, a una retórica de crecimiento de los límites, que los considera impulsores de acumulación, "filtros" que convierten el constreñimiento ecológico en una crisis por el capitalismo (Pellizzoni, 2018).

Además, es posible argüir que los bienes comercializados en los mercados ambientales contienen valor, dado que son producidos por unidades híbridas de trabajo (reproductivo/ informativo) y por la naturaleza (financializada) (Leonardi, 2019). Sin embargo, el potencial de desarrollo de esta economía verde postdualista debe ser relativizado. No sólo porque "el hecho de que el capital sienta cada límite como un obstáculo y luego trate de superarlo idealmente no deriva del hecho que aquel límite sea realmente superado" (Marx, 20I2, p. 274), sino también y sobre todo porque el proceso de potenciar la actividad "gratuita" de la naturaleza no parece, por lo menos hasta el momento, lograr subsanar el daño medioambiental ya hecho o proporcionar protecciones sociales generalizadas que permitan compensar la polarización de clases asociada la multiplicación de los dividendos financieros.

El capitalismo neoliberal carece — repetita iuvant, al menos hasta el momento- de un mecanismo inclusivo capaz de socializar (parcialmente) los beneficios financieros a través de la descarbonización de la economía o la formación de una nueva clase media (o ambos). Un buen camino para mejor comprender esta fricción es indicado por André Gorz, que, en una de sus más recientes entrevistas, define la creciente ruptura entre el valor y la riqueza del siguiente modo:

"Producir más y más no es pues un problema. El problema es la creciente distancia entre la capacidad de producir y la

\footnotetext{
Se ha criticado la ontología "híbrida" (es decir, no dualista) de Moore por ser filosóficamente defectuosa y desprovista de poder político (Malm, 20l8). Es una crítica legítima que merece analizarse detenidamente. Con todo, ese análisis nos desviaría demasiado de los objetivos de este artículo. Lo que quisiera destacar aquí es la relación entre la explotación y la naturaleza. Moore está en lo cierto al defender que, para que funcionara correctamente, la explotación ha exigido históricamente que la naturaleza fuera "capitalizada" como un contexto externo. Esto, sin embargo, parece ya no poderse aplicarse (completamente) si consideramos las características del proceso de creación de valor (por ende, explotación) que ocurre en una variedad de mercados presuntamente "ambientales" y, en particular, en la comercialización de los derechos de emisión de carbono (Leonardi, 20l7b).
} 
capacidad de vender con beneficio, entre la riqueza producible y la forma mercancía, la forma de valor que la riqueza debe obligatoriamente revestir para poder ser producida en el marco del sistema económico vigente" (Gorz, 2008, p. I36).

De esta fricción — que se ha profundizado también desde una perspectiva ecológica y, por eso, no ha sido abordada en los últimos años por los investigadores vinculados al operaísmo (Vercellone et al., 2017) — no deriva la imposibilidad de mercantilizar la naturaleza en formas distintas de las típicamente dualistas que caracterizaron la modernidad hasta la decadencia del fordismo. De ella procede la dificultad del capitalismo neoliberal para construir una arquitectura institucional con capacidad para producir beneficios financieros y redistribución destinada a promover el consenso social. Sencillamente, nos quedamos con una antigua certidumbre presentada con ropajes distintos: "que el capitalismo no colapsa mecánicamente ni procede de sí mismo en el ámbito de su proceso de evolución, y, por ende, su superación no es concebible sino a partir de una intervención política" (Napoleoni, 1970, p. LXX).

Es evidente que la forma que tomará esta intervención deberá tener en cuenta tanto los mecanismos de valoración del capital globalizado como las relaciones entre clases que en él se establecen. El análisis de este último aspecto carecería de un artículo adicional para completarse. No obstante, es posible indicar tres líneas de investigación futura. En primer lugar, desplazar del centro del debate el aspecto sobre el desenlace de la crisis —evitando así "la inclinación hacia el colapso" o "la inclinación hacia el desarrollo"- permitiría que temas de análisis innovadores centrasen su atención teórico-práctica en los elementos deseables de la transición ecológica. En segundo lugar, es necesario cuestionar la relación entre el carácter local de los conflictos ambientales y la escala global de los efectos producidos por la crisis — como el cambio climático-, tendiendo un puente entre la autonomía de base comunitaria y la configuración de la ecología como un espacio más amplio de recomposición política (Torre, 2017). Finalmente, será decisivo establecer un vínculo inextricable entre la transición ecológica de las infraestructuras económicas y las luchas de clases del siglo XXI: se tratan de dos caras de la misma moneda, dado que la lucha contra la desigualdad constituye el primer objetivo de cualquier conflicto ambiental digno de ese nombre.

\section{Referencias}

Andreucci, D., et al. (2017). 'Value Grabbing': A Political Ecology of Rent. Capitalism Nature Socialism, 28 (3), $28-47$.

Armiero, M. (202I). Wastocene. Cambridge University Press.

Avallone, G. (2015). Introduzione. En Moore J. Ecologia-mondo e crisi del capitalismo. La fine della natura a buon mercato. Ombre corte.

Balestrini, N. y Moroni, P. (2005). L'orda d'oro 1968-1977. Feltrinelli.

Barca, S. (20I I). Pane e veleno. Storie di ambientalismo operaio in Italia. Zapruder, 24, 99-I 05.

Barca, S. (2020). Forces of Reproduction. Cambridge University Press.

Benegiamo, M. (202I). Exploring Accumulation in the New Green Revolution for Africa: Ecological Crisis, Agrarian Development and Bio-Capitalism. En Benquet, M. y Bourgeron, T. (Eds.). Accumulating Capital Today : Contemporary Strategies of Profit and Dispossessive Policies (6I-74). Routledge.

Bellofiore, R. ( I 982). L'operaismo degli anni '60 e la critica dell'economia politica. Unità proletaria, I-2, I00-I I 2.

Bellofiore, R. (1988). Il rosso, il rosa e il verde. Considerazioni inattuali su centralità operaia e nuovi movimenti. Quaderni del CRIC, 3, 7-29.

Bellofiore, R. (20I2). La crisi capitalistica, la barbarie che avanza.Asterios.

Bologna, S. (1988). Emarginazione e ambientalismo. Primo Maggio, 27-28, 35-39. 
Chignola, S. (20I5). Italian Theory? Elementi per una genealogia. En Gentili, D. y Stimilli, E. Differenze italiane. Deriveapprodi.

Citoni, M. y Papa, C. (2017). Sinistra ed ecologia in Italia, 1968-1974. Micheletti.

Cleaver, H. (1972). The Contradictions of the Green Revolution. American Economic Review, 62 (2), I73- 179.

Colletti, L. (1970). Introduzione. En Colletti, L. y Napoleoni, C. Il futuro del capitalismo: crollo o sviluppo? Laterza.

Davigo, E. (2017). II movimento italiano per la tutela della salute negli ambienti di lavoro (I96/-1978). Tesi di dottorato, Università di Firenze.

Della Valentina, G. (20I I). Storia dell'ambientalismo in Italia. Bruno Mondadori.

Fairbairn, M. (20I4). 'Like gold with yield': evolving intersections between farmland and finance. The Journal of Peasant Studies, 4I (5), 777-795.

Foster, J.B. (20I I). Capitalism and the Accumulation of Catastrophe. Monthly Review, 63 (7).

Felli, R. (20I4). On Climate Rent. Historical Materialism, 22 (3-4), 25 I-280.

Feltrin, L. y Sacchetto, D. (202I). The Work-Technology Nexus and Working-Class Environmentalism: Workerism versus Industrial Toxicity in Italy's Long 1968. Theory and Society.

Filippini, M.y Tomasello, F. (20 I0). Il pensiero come arnese: note sul metodo operaista degli anni Sessanta. En Simoncini, A. Dal pensiero critico: filosofie e concetti per il tempo presente. Mimesis.

Fumagalli, A. y Mezzadra, S. (2009). Crisi dell'economia globale. Ombre corte.

Fumagalli, A., Giuliani, A., Lucarelli, S. y Vercellone, C. (2019). Cognitive Capitalism, Welfare and Labour: the Commonfare Hypothesis. Routledge.

Gentili, D. (20I8). La crisi come arte di governo. Quodlibet.

Gorz,A. (2008). Ecologica. Jaca Book.

Grossman, H. (2010). Il crollo del capitalismo. Mimesis.

Hardt, M. y Negri, A. (20I8). Assemblea. Ponte alle Grazie.

Inglehart, R. (1997). Valori e cultura politica nella società industriale avanzata. UTET.

Leonardi, E. (2017a). Lavoro Natura Valore. Orthotes.

Leonardi, E. (20I7b). Carbon Trading Dogma:Theoretical Assumptions and Practical Implications of Global Carbon Markets. Ephemera, I7 (I), 6 I-87.

Leonardi, E. (2019). Bringing Class Back In: assessing the transformation of the value-nature nexus to strengthen the connection between degrowth and environmental justice. Ecological Economics, 156, 83-90.

Leonardi, E. y Pellizzoni, L. (Eds.) (2019). Italian Perspectives on World-Ecology. Sociologia Urbana e Rurale, I 20 (special issue).

Lucarelli, S. (2009). La finanziarizzazione come forma di biopotere. En Fumagalli, A. y Mezzadra S. Crisi dell'economia globale. Ombre corte.

Luxemburg, R. (20I2). L'accumulazione del capitale. Pgreco.

Marx, K. ( I 973). II Capitale (vol. III). Editori Riuniti.

Marx, K. (20I2). Grundrisse. Manifestolibri.

Malm,A. (2018). The Progress of This Storm. Verso.

Marzocca, O. (1998). Transizioni senza meta. Mimesis.

Milanaccio, A. y Ricolfi, L. (1976). Lotte operaie e ambiente di lavoro: Mirafiori 1968-1974. Einaudi.

Moore, J. (20I4). The End of Cheap Nature. Or How I Learned to Stop Worrying about "The" Environment and Love the Crisis of Capitalism. En Suter, C.y Chase-Dunn, c. (Eds.). Structures of the World Political Economy and the Future of Global Conflict and Cooperation (pp. 285-3।4). LIT.

Moore, J. (20I5). Capitalism in the Web of Life.Verso.

Negri, A. (1972). Marx sul ciclo e la crisi. En AAVV. Operai e Stato. Feltrinelli.

Negri,A. (2009). Qualche riflessione sulla rendita dentro la grande crisi. En Fumagalli A.y Mezzadra S. Crisi dell'economia globale. Ombre corte.

O'Connor, J. (1988). Capitalism, Nature, Socialism: a Theoretical Introduction. Capitalism Nature Socialism, I.

Pellizzoni, L. (2018). The commons in the shifting problematization of contemporary society. Rassegna italiana di sociologia, LIX (2), 2 I I-233.

Pellizzoni, L. (2019). Ontological Politics in a Disposable World. Ashgate.

Rector, J. (20I4). Environmental Justice at Work. Journal of American History, I0I (2), 480-502. https://doi.org/I0.1093/ jahist/jau380

Ronchi, E., (20 18). Relazione sullo stato della Green Economy. Fondazione per lo sviluppo sostenibile.

Roth, K.H. (2009). Esterni e interni: l'autonomia operaia di Porto Marghera vista dalla Germania Ovest. En Sacchetto, D. y Sbrogiò, G. Quando il potere è operaio. Manifestolibri.

Sacchetto, D. y Sbrogiò, G. (2009). Quando il potere è operaio. Manifestolibri.

Torre, S. (2017). Contro la frammentazione. Ombre corte.

Torre, S. (2020). II metodo del vivente. Geography Notebooks, 3, 20 I-2I 5.

Trotta, G. y Milana, F. (2008). L'operaismo degli anni Sessanta. Deriveapprodi.

Vercellone, C., Brancaccio, F., Giuliani, A. y Vattimo, P. (20I7). II comune come modo di produzione. Ombre corte.

Wright, S. (2017). Storming Heaven. Pluto Press. 


\section{RELACIONES INTERNACIONALES}

Revista académica cuatrimestral de publicación electrónica

Grupo de Estudios de Relaciones Internacionales (GERI)

Universidad Autónoma de Madrid, España

https://revistas.uam.es/relacionesinternacionales

ISSN 1699 - 3950

f facebook.com/RelacionesInternacionales

3. twitter.com/RRInternacional 